ARTICLE

DOI: $10.1038 / s 41467-018-07177-y$

\title{
Atomic scale insights into structure instability and decomposition pathway of methylammonium lead iodide perovskite
}

Shulin Chen ${ }^{1,2,3}$, Xiaowei Zhang ${ }^{3}$, Jinjin Zhao ${ }^{4}$, Ying Zhang ${ }^{4}$, Guoli Kong ${ }^{4}$, Qian Li (1) ${ }^{5}$, Ning Li, ${ }^{1,3}$ Yue $\mathrm{Yu}^{6}$, Ningan $\mathrm{Xu}^{7}$, Jingmin Zhang ${ }^{1}$, Kaihui Liu (1) ${ }^{8,9}$, Qing Zhao ${ }^{8,9}$, Jian Cao ${ }^{2}$, Jicai Feng ${ }^{2}$, Xinzheng $\mathrm{Li}^{8,9}$, Junlei Qi ${ }^{2}$, Dapeng Yu (1,8,9,10, Jiangyu Li ${ }^{11,12}$ \& Peng Gao (1) 1,2,3,9

Organic-inorganic hybrid perovskites are promising candidates for the next-generation solar cells. Many efforts have been made to study their structures in the search for a better mechanistic understanding to guide the materials optimization. Here, we investigate the structure instability of the single-crystalline $\mathrm{CH}_{3} \mathrm{NH}_{3} \mathrm{Pbl}_{3}\left(\mathrm{MAPbl}_{3}\right)$ film by using transmission electron microscopy. We find that $\mathrm{MAPbl}_{3}$ is very sensitive to the electron beam illumination and rapidly decomposes into the hexagonal $\mathrm{Pbl}_{2}$. We propose a decomposition pathway, initiated with the loss of iodine ions, resulting in eventual collapse of perovskite structure and its decomposition into $\mathrm{Pbl}_{2}$. These findings impose important question on the interpretation of experimental data based on electron diffraction and highlight the need to circumvent material decomposition in future electron microscopy studies. The structural evolution during decomposition process also sheds light on the structure instability of organic-inorganic hybrid perovskites in solar cell applications.

\footnotetext{
${ }^{1}$ Electron Microscopy Laboratory, School of Physics, Peking University, Beijing 100871 , China. ${ }^{2}$ State Key Laboratory of Advanced Welding and Joining, Harbin Institute of Technology, Harbin 150001, China. ${ }^{3}$ International Center for Quantum Materials, School of Physics, Peking University, Beijing 100871, China. ${ }^{4}$ School of Materials Science and Engineering, Shijiazhuang Tiedao University, Shijiazhuang 050043, China. ${ }^{5}$ X-ray Science Division, Argonne National Laboratory, Lemont, IL 60439, USA. ${ }^{6}$ Center for Nanochemistry, College of Chemistry and Molecular Engineering, Peking University, Beijing 100871, China. ${ }^{7}$ Oxford Instruments Technology (Shanghai) Co. Ltd., Shanghai 200233, China. ${ }^{8}$ State Key Laboratory for Mesoscopic Physics, School of Physics, Peking University, Beijing 100871, China. ${ }^{9}$ Collaborative Innovation Center of Quantum Matter, Beijing 100871, China. ${ }^{10}$ Department of Physics, South University of Science and Technology of China, Shenzhen 518055, China. ${ }^{11}$ Shenzhen Key Laboratory of Nanobiomechanics, Shenzhen Institutes of Advanced Technology, Chinese Academy of Sciences, Shenzhen 518055 Guangdong, China. ${ }^{12}$ Department of Mechanical Engineering, University of Washington, Seattle, WA $98195-$ 2600, USA. These authors contributed equally: Shulin Chen, Xiaowei Zhang. Correspondence and requests for materials should be addressed to J.Z. (email: jinjinzhao2012@163.com) or to J.Q. (email: jlqi@hit.edu.cn) or to J.L. (email: jjli@uw.edu) or to P.G. (email: p-gao@pku.edu.cn)
} 
S olar cells based on organic-inorganic hybrid perovskites $\left(\mathrm{CH}_{3} \mathrm{NH}_{3} \mathrm{PbI}_{3}, \quad \mathrm{MAPbI}_{3}\right)$ have attracted widespread research attention due to their low synthesis cost and highpower conversion efficiency $(\mathrm{PCE})^{1-3}$. Despite the great progress in increasing the device PCE from initial $3.8 \%{ }^{4}$ to the most recent $23.3 \%{ }^{5}$ in just one decade, the commercialization of this technology remains hindered by the stability issues of materials. It has been reported that the perovskites rapidly degrade under increased temperature ${ }^{6}$, oxygen ${ }^{7}$, moisture $^{8}$, and UV light illumination ${ }^{9}$, often due to the structure instability caused by electromigrations ${ }^{10}$, ion migration, ${ }^{11}$ and interfacial relationships ${ }^{12}$. In fact, with increasing temperature, visible diffusion of iodine initiates at a temperature below $150^{\circ} \mathrm{C}$ and lead migration is induced at a higher temperature around $175^{\circ} \mathrm{C}$, causing the degradation of perovskite and formation of $\mathrm{PbI}_{2}{ }^{6}$. The ion migration induced by the electric field, heat, and light illumination also contributes to the hysteresis in $\mathrm{J}-\mathrm{V}$ curves $^{13,14}$, which in turn leads to poor long-term stability resulted from significant structural changes, including the lattice distortion and the phase decomposition. Thus, it is essential to characterize the intrinsic structure as well as structure stability and the decomposition pathway of these materials to improve organic-inorganic hybrid perovskites, which motivates our present transmission electron microscopy (TEM) study on perovskite $\mathrm{MAPbI}_{3}$.

Although TEM has been widely used for structure characterization of perovskite $\mathrm{MAPbI}_{3}{ }^{15-17}$, the atomically resolved imaging remains elusive due to its electron beam-sensitive nature ${ }^{18}$. Thus, the electron diffraction (ED) or fast Fourier transform (FFT) pattern based on lattice fringes becomes a common preference to identify the phase of perovskite. However, in most of the experiments, the observed ED or FFT patterns do not really match those of a perfect tetragonal perovskite phase exactly $16,17,19-23$. For example, $\{110\}$ diffraction spots along the [001] direction are usually not observed in electron microscopy even though these reflections are visible from X-ray diffraction (XRD) measurements ${ }^{24-29}$. Long et al. ${ }^{24}$ identified a textured thin $\mathrm{MAPbI}_{3}$ film to be a tetragonal [001] zone axis even though $\{110\}$ spots were not observed. Park et al. ${ }^{25}$ prepared a cross-sectional whole device of glass/FTO/mp-TiO $/ \mathrm{MAPbI}_{3} /$ spiro-OMeTAD, and similarly the $\{110\}$ spots were absent. Polarz et al. ${ }^{26}$ developed metal-organic porous single-crystalline $\mathrm{MAPbI}_{3}$ and also did not observe the (1 10) spots. Furthermore, Tang et al. ${ }^{27}$, Vela et al. ${ }^{28}$, and Zhu et al. ${ }^{29}$ found that the $\{110\}$ spots were missing in $\mathrm{MAPbI}_{3}$ nanowires. Segawa $^{30}$ explained that the $(110)$ and (110) diffraction spots are inherently difficult to be detected experimentally due to the low ED intensity of organic-inorganic halide compounds. Despite the absence of some expected diffraction spots, the corresponding structures were often identified as a tetragonal perovskite phase, as summarized in Supplementary Table 1. Nevertheless, $\{110\}$ diffraction spots of perovskite ${ }^{31}$ have been captured from a rapid acquisition with the dose rate as low as 1 e $\AA^{-2} s^{-1}$, suggesting that the absence of $\{110\}$ reflections of perovskite may be related to structure degradation during electron microscopy characterizations. From an energetic point of view, electron beam illumination, optical illumination, heating, and the electric field are similar, enabling ions to overcome the migration barriers and thus inducing the structure transition ${ }^{32-34}$. Revealing the structure evolution of $\mathrm{MAPbI}_{3}$ under electron illumination, especially its decomposition pathway, therefore, can help us to understand material degradation in practical solar cell devices.

In this paper, we use atomically resolved Z-contrast imaging, diffraction analysis, and quantitative energy- dispersive X-ray spectroscopy (EDX) in an aberration-corrected TEM in an attempt to understand the structure and structure evolution of single-crystalline tetragonal $\mathrm{MAPbI}_{3}$. We found that the perovskite structure undergoes complex phase changes under the electron beam illumination. The decomposition of $\mathrm{MAPbI}_{3}$ is likely initiated with the loss of I ions to subsequently form superstructure $\mathrm{MAPbI}_{2.5}$, followed by the loss of MA and additional I ions to form $\mathrm{MA}_{y} \mathrm{PbI}_{2.5-z}(0 \leq y \leq 1$ and $0 \leq z \leq 0.5)$ accompanied with the disappearance of a superstructure, leading to its final decomposition into $\mathrm{PbI}_{2}$. During this process, the ED patterns change subtly that is often overlooked, and thus the evolving and decomposed phases are often incorrectly identified as perovskite. Indeed, if we can only observe $\{2 \mathrm{~h}, 2 \mathrm{k}, 0\}$ diffraction spots along the [001] direction and while the $\{2 \mathrm{~h}+1,2 \mathrm{k}+1,0\}$ reflections [e.g., (110)] are absent, it is highly likely that the tetragonal perovskite phase has already decomposed into a hexagonal $\mathrm{PbI}_{2}$ structure along the $[\overline{4} 41]$ zone axis. We also identify the critical electron dose limit to obtain the intrinsic perovskite structure. Our findings impose important questions on some earlier experimental interpretations of ED measurements of $\mathrm{MAPbI}_{3}$ and highlight the need to circumvent material degrading in future experiments. The identified optimal TEM conditions to obtain the intrinsic perovskite structure can be used to guide the future electron microscopy characterization of these materials. The understanding of the degradation process also sheds light into the general stability issue of the organic-inorganic hybrid perovskites in solar cell applications.

\section{Results}

Mismatches in ED patterns. $\mathrm{MAPbI}_{3}$ crystals were self-grown on FTO/TiO 2 substrates, as reported in our previous study ${ }^{35,36}$. The powder XRD (Supplementary Fig. 1a) indicates a good crystallinity of tetragonal $\mathrm{MAPbI}_{3}{ }^{37,38}$. The single-crystalline nature is further confirmed by synchrotron X-ray diffraction (XRD) (Supplementary Fig. 1b) with the measured $(004)_{c}$ reflection denoted in the pseudo-cubic setting. However, when $\mathrm{MAPbI}_{3}$ crystal was put into TEM, the acquired ED and FFT patterns do not match those of a perfect tetragonal perovskite structure. The atomistic models of tetragonal $\mathrm{MAPbI}_{3}$ along [100] and [001] axis are shown in Fig. 1a, b, and the corresponding ED patterns simulated from the perfect structure are shown in Fig. 1c, e, and g. A subtle difference between the experimental SAED (Fig. 1d and $h$ ) and FFT pattern (Fig. 1f) acquired from regions in Supplementary Fig. 2 and the simulated ones can be noted after careful comparison. For example, Fig. 1d is identified to be the [001] zone axis of tetragonal $\mathrm{MAPbI}_{3}$, which is similar to the simulated ED pattern in Fig. 1c, yet $\{110\}$ diffraction spots do become dimmer. More importantly, Fig. 1f is identified to be the [210] zone axis, wherein the (002) diffraction spot can hardly be seen. Figure $1 \mathrm{~h}$ is identified to be the $[10 \overline{1}]$ zone axis, wherein the (020) spot is not observed while an additional (101) spot appears. Therefore, whether these SAED patterns indeed correspond to tetragonal $\mathrm{MAPbI}_{3}$ is questionable and needs further investigation.

Atomic structure of $\mathbf{P b I}_{2}$. To resolve the subtle difference between experimental and simulated ED patterns, atomically resolved Z-contrast images were recorded at $80 \mathrm{kV}$. An atomically resolved STEM image in Fig. 2a shows stripes with a measured distance of $0.72 \mathrm{~nm}$. Based on the corresponding FFT pattern (Fig. 2b), the viewing direction is often mistaken as the [101] of $\mathrm{MAPbI}_{3}$ without noticing that additional (101) reflection appears while the (020) spot is missing. After careful analysis, we found that these stripes are in fact not from $\mathrm{MAPbI}_{3}$ but $\mathrm{PbI}_{2}$, which can be illustrated by the ball-and-stick model of the $\mathrm{PbI}_{2}$ (Fig. 2c). Furthermore, simulated HAADF-STEM (Fig. 2e) was carried out to compare with the experimental image contrast (Fig. 2a), which shows good agreement. The experimental FFT pattern (Fig. 2b) can be identified to be the [120] direction of $\mathrm{PbI}_{2}$, which matches 

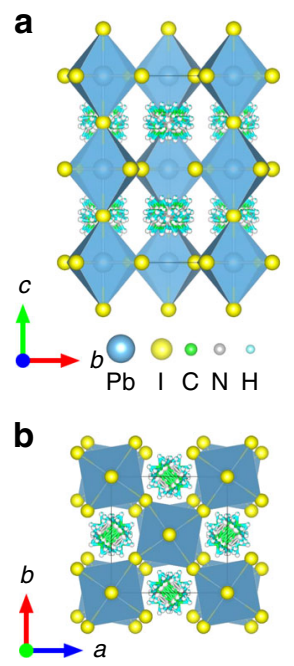

e
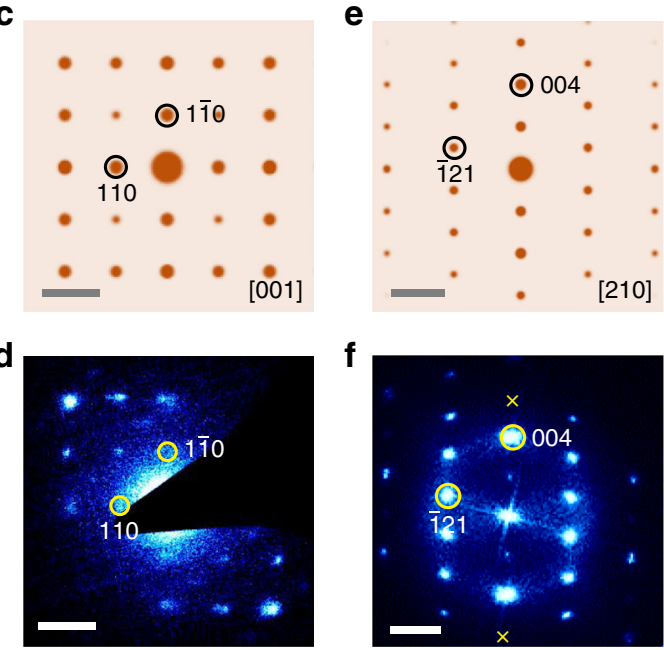

g
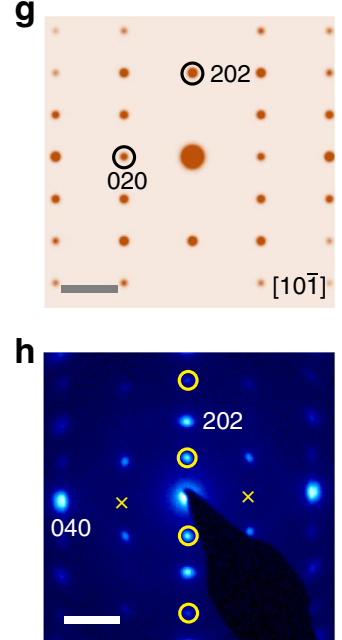

Fig. 1 The atomic structure of tetragonal $\mathrm{MAPb}_{3}$. The atomic models of tetragonal $\mathrm{MAPbl}_{3}$ along $\mathbf{a}[100]$ and $\mathbf{b}$ [001] zone axis. The simulated electron diffraction (ED) patterns of tetragonal $\mathrm{MAPb}_{3}$ along $\mathbf{c}$ [001], $\mathbf{e}$ [210], and $\mathbf{g}$ [101] zone axes, respectively. $\mathbf{d}, \mathbf{h}$ The selected area electron diffraction (SAED) and $\mathbf{f}$ fast Fourier transform (FFT) pattern of tetragonal $\mathrm{MAPbl}_{3}$. Note that there is a subtle difference between the experiment and simulation. The $\{110\}$ diffraction spots appear to be dimmer in $\mathbf{d}$, and the (002) spot almost vanishes and the (006) spot disappears marked by yellow crosses in $\mathbf{f}$, and the (020) spot is not observed marked by yellow crosses but additional $\{2 \mathrm{~h}+1,0,2 \mathrm{~h}+1\}$ reflections [e.g., (101)] appear indicated by yellow circles in $\mathbf{h}$. Scale bar, $2 \mathrm{~nm}^{-1}(\mathbf{c}-\mathbf{h})$

the simulated ED pattern (Fig. 2d) well. The detailed atomistic configurations of the hexagonal $\mathrm{PbI}_{2}$ (space group: $\mathrm{R}-3 \mathrm{~m}: \mathrm{H}, a=$ $b=0.4557 \mathrm{~nm}, c=2.0937 \mathrm{~nm}, \alpha=\beta=90^{\circ}$, and $\gamma=120^{\circ}$ ) are shown in Supplementary Fig. $3^{39}$, wherein the layered $\mathrm{PbI}_{2}$ structure is composed of $\mathrm{Pb}-\mathrm{I}$ octahedra. The stability of this structure at room temperature is further confirmed by the molecular dynamics (MD) simulation at $300 \mathrm{~K}$.

Additional atomic structure data along different directions (Fig. 2f-t) further confirm that the original sample of $\mathrm{MAPbI}_{3}$ is indeed decomposed into $\mathrm{PbI}_{2}$. These STEM images have been filtered and the pristine images are shown in Supplementary Fig. 4. Figure $2 \mathrm{f}$ shows the alternating $\mathrm{Pb}$ and $\mathrm{I}$ arrangements of $\mathrm{PbI}_{2}$, illustrated by the ball-and-stick model in Fig. 2h. The FFT pattern (Fig. 2g) from the region (Supplementary Fig. 4b) is identified to be the $\left[\begin{array}{lll}8 & 10 & 1\end{array}\right]$ zone axis, which is consistent with simulated ED pattern (Fig. 2i). In addition, the simulated STEM image in Fig. $2 \mathrm{j}$ also shows a similar contrast, compared with Fig. 2f. Likewise, atomic STEM images along [111] and [110] zone axes have also been analyzed as shown in Fig. $2 \mathrm{k}-\mathrm{t}$. These STEM images well match the corresponding ball-and-stick models and simulated STEM images of the $\mathrm{PbI}_{2}$, and the corresponding FFT patterns are also consistent with the simulated ED patterns of the $\mathrm{PbI}_{2}$. Additional data of $\mathrm{PbI}_{2}$ are presented in Supplementary Fig. 5, showing a subtle difference with simulated $\mathrm{MAPbI}_{3}$ in the ED pattern in Supplementary Fig. 6. It is worth noting that both ED patterns of [ $\left[\begin{array}{lll}8 & 10 & 1\end{array}\right]$ and [ 111] zone axes of $\mathrm{PbI}_{2}$ are often easily mistaken for the [101] direction of $\mathrm{MAPbI}_{3}$ when the missing of (020) diffraction spot from $\mathrm{MAPbI}_{3}$ is not recognized.

Structural comparison between $\mathrm{MAPbI}_{3}$ and $\mathbf{P b I}_{2}$. It is quite revealing to highlight the difference in atomic structures and $\mathrm{ED}$ patterns between $\mathrm{MAPbI}_{3}$ and $\mathrm{PbI}_{2}$. Figure $3 \mathrm{a}$ is a HAADF-STEM image of $\mathrm{PbI}_{2}$ viewing along the [441] direction judging from the corresponding FFT pattern (Fig. 3b) as well as the SAED and TEM images (Supplementary Fig. 7). The atomistic model, simulated ED pattern, and simulated STEM image of $\mathrm{PbI}_{2}$ along this zone axis are shown in Fig. $3 \mathrm{c}-\mathrm{e}$. However, in many previous studies $^{16,17,19-29}$, this ED or FFT pattern has been mistakenly identified as [001] zone axis of tetragonal $\mathrm{MAPbI}_{3}$ without noticing the absence of $\{110\}$ diffraction spots (Supplementary Table 1). For comparison, based on the atomistic model in Fig. 3f, we also carried out the simulations of ED (Fig. 3g) and HAADF image (Fig. 3h) for $\mathrm{MAPbI}_{3}$ along the [001] zone axis. Note that the distance in reciprocal space for (220) reflection of tetragonal $\mathrm{MAPbI}_{3}$ is $0.3196 \AA^{-1}$ (Fig. $3 \mathrm{~g}$ ), which is extremely close to that of (104) and $(0 \overline{1} 4)$ reflections $\left(0.3173 \AA^{-1}\right)$ for $\mathrm{PbI}_{2}$ (Fig. $3 \mathrm{~d}$ ), making it difficult to distinguish them only based on the SAED pattern. Despite of the similarity in their ED patterns, the simulated atomic structures of these two phases are quite different. For tetragonal $\mathrm{MAPbI}_{3}$ along the [001] zone axis, the contrast of atom columns is not uniform (Fig. $3 \mathrm{~h}$ ), in comparison with the uniform contrast in $\mathrm{PbI}_{2}$ along [441] in Fig. 3e. Moreover, the distance between two brighter atom columns is $0.625 \mathrm{~nm}$ for $\mathrm{MAPbI}_{3}$ (Fig. 3h), which is twice the distance between two brighter atom columns $(0.315 \mathrm{~nm})$ of $\mathrm{PbI}_{2}$ (Fig. 3e).

The structure evolution during decomposition. The evolution of tetragonal $\mathrm{MAPbI}_{3}$ decomposing into hexagonal $\mathrm{PbI}_{2}$ is important for understanding the material degradation, and it can be captured at a low magnification with SEM and a series of EDX mappings (Fig. 4). During the process, the $\mathrm{I} / \mathrm{Pb}$ atom ratio decreases from the initial 2.62 to the final 1.92 due to the volatilization of the iodine $\mathrm{e}^{40,41}$ (Supplementary Fig. 8), consistent with the formation of $\mathrm{PbI}_{2}$. The quantitative EDX mapping in the STEM mode at $80 \mathrm{kV}$ also shows that the average atom ratio for I/ $\mathrm{Pb}$ is close to 2 (Supplementary Table 2, based on the spectrum in Supplementary Fig. 9). Additional STEM-EDX mapping was conducted on other samples (Supplementary Fig. 10) showing similar observations.

In order to capture the intrinsic perovskite structure and investigate the detailed degradation process, the evolution of ED patterns under a low-dose rate $\sim 1$ e $\AA^{-2} \mathrm{~s}^{-1}$ is recorded in Fig. 5a-d viewing along [001] $\mathrm{MA}_{y} \mathrm{PbI}_{3-x}(0 \leq y \leq 1$ and $0 \leq x \leq 1)$ (or $[\overline{4} 41]$ of $\mathrm{PbI}_{2}$ ). These time-series SAED patterns match the corresponding simulated ones (Fig. $5 \mathrm{e}-\mathrm{h}$ ) based on the atomistic structures (Fig. 5i-l). During the decomposition, we observe an intermediate phase with superstructure spots (Fig. 5b), which can 

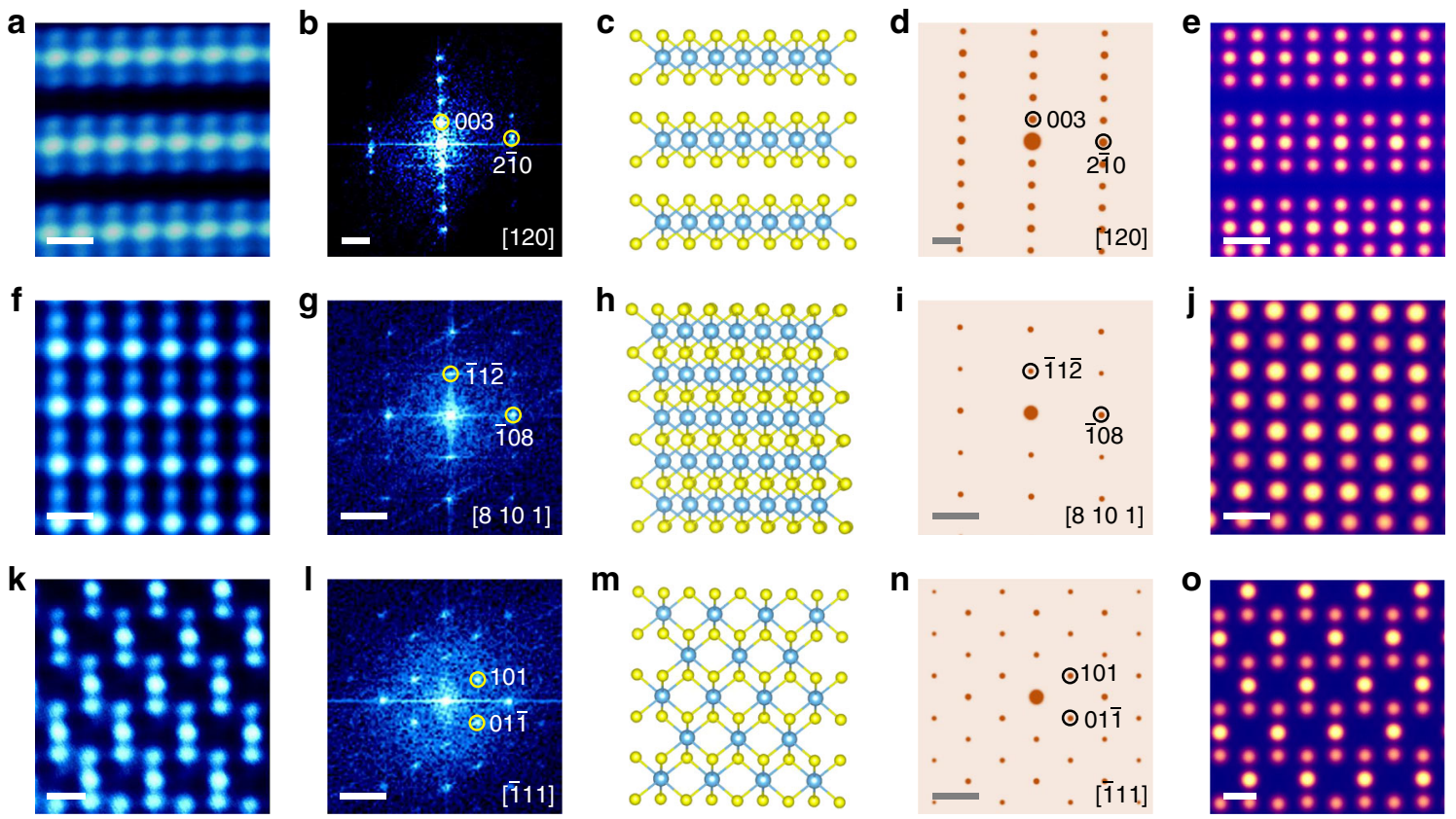

n
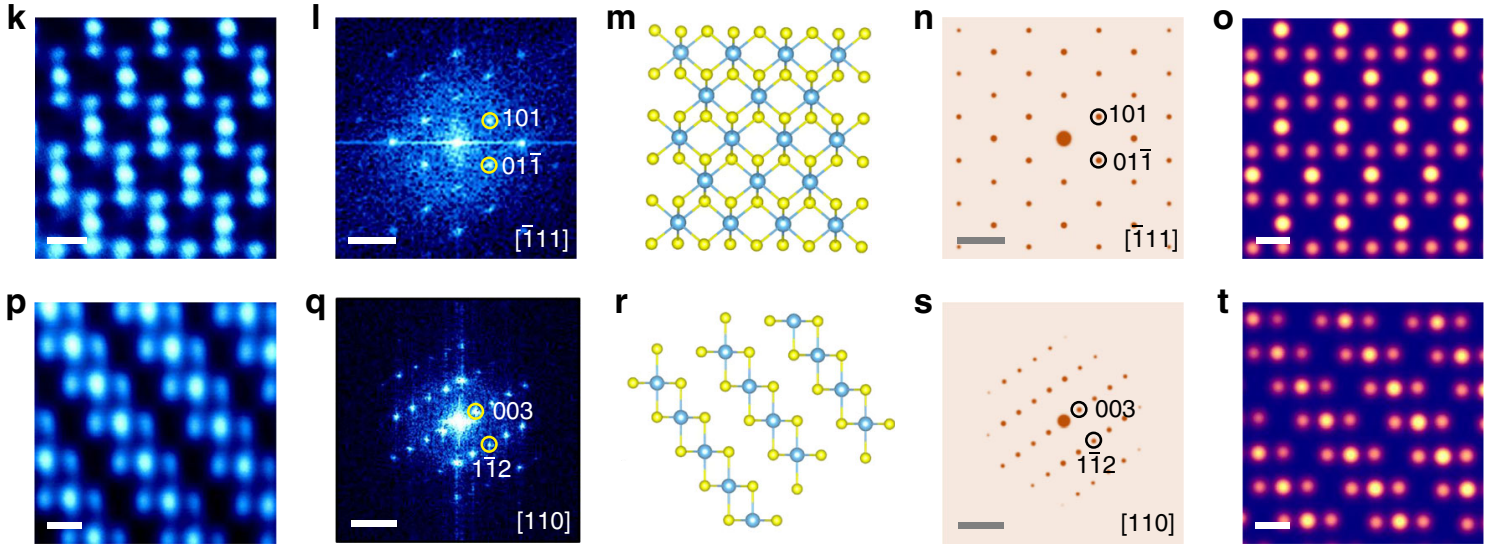

$\mathbf{S}$

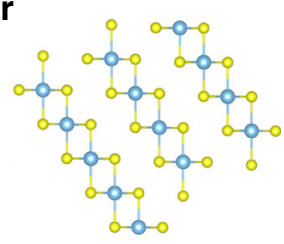

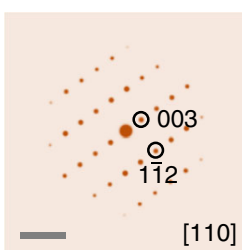

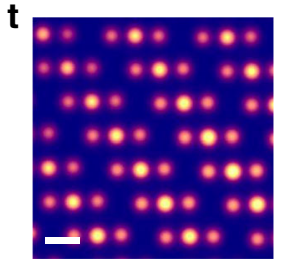

Fig. 2 The atomic structure of $\mathrm{Pbl}_{2}$ along different zone axes. $\mathbf{a}, \mathbf{f}, \mathbf{k}, \mathbf{p}$ STEM images of $\mathrm{Pbl}_{2}$ along [120], [8 10 1], [111], and [110] zone axes with $\mathbf{b}, \mathbf{g}, \mathbf{I}, \mathbf{q}$ the corresponding FFT patterns, $\mathbf{c}, \mathbf{h}, \mathbf{m}, \mathbf{r}$ atomic ball-and-stick models, $\mathbf{d}, \mathbf{i}, \mathbf{n}, \mathbf{s}$ simulated ED patterns, and $\mathbf{e}, \mathbf{j}, \mathbf{0}, \mathbf{t}$ simulated STEM images. Scale bar, $0.3 \mathrm{~nm}(\mathbf{a}, \mathbf{e}, \mathbf{f}, \mathbf{j}, \mathbf{k}, \mathbf{0}, \mathbf{p}, \mathbf{t}) ; 2 \mathrm{~nm}^{-1}(\mathbf{b}, \mathbf{d}) ; 3 \mathrm{~nm}^{-1}(\mathbf{g}, \mathbf{i}, \mathbf{l}, \mathbf{n}, \mathbf{q}, \mathbf{s})$

be fitted by $\mathrm{MAPbI}_{2.5}$ with ordered iodine vacancies as shown in Fig. 5j. Compared with the pristine $\mathrm{MAPbI}_{3}$ in Fig. 5i, part of iodine ions on the (001) plane indicated by the black circles in Fig. $5 \mathrm{j}$ are lost to form the superstructure $\mathrm{MAPbI}_{2.5}$. The corresponding simulated $\mathrm{ED}$ pattern of $\mathrm{MAPbI}_{2.5}$ in Fig. $5 \mathrm{f}$ matches well with the experimental result in Fig. 5b. Furthermore, from another viewing direction of [100] of perovskite, the ED pattern corresponding to superstructure $\mathrm{MAPbI}_{2.5}$ is also observed (see details in Supplementary Fig. 11). In fact, iodine ions are the most likely mobile ions due to the lower diffuse barrier in $\mathrm{MAPbI}_{3}{ }^{11,42,43}$, similar to the oxygen ions in the oxide perovskites ${ }^{44}$, and the migration of iodine ions in $\mathrm{MAPbI}_{3}$ indeed has been reported from the previous studies ${ }^{10,14,45}$. Thus, we infer that the superstructure is likely caused by the formation of ordered I vacancies. Moreover, forming such an intermediate superstructure $\mathrm{MAPbI}_{2.5}$ tends to minimize the repulsive interaction between iodine ions as well as the elastic coherence strain energy, similar to the staging phases in the lithium ion battery materials ${ }^{46,47}$.

With further increased electron beam dose, the structure continues to lose MA and additional I ions to form $\mathrm{MA}_{y} \mathrm{PbI}_{2.5-z}$ $(0 \leq y \leq 1$ and $0 \leq z \leq 0.5)$ (Fig. $5 \mathrm{k}$ ), which is accompanied by the disappearance of the superstructure. Finally, the perovskite structure framework collapses to form the layered $\mathrm{PbI}_{2}$ structure (Fig. 5l). Note that the ED pattern in Fig. $5 \mathrm{c}$ can be regarded as a

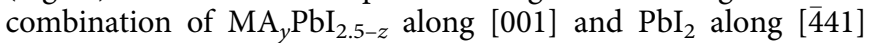
directions, consistent with the overlap of the corresponding simulated ED patterns in Fig. 5g (see details in Supplementary Fig. 12). It is also interesting to note that the previous study has proposed the reaction pathway from $\mathrm{PbI}_{2}$ to $\mathrm{MAPbI}_{3}$ during synthesis ${ }^{48}$, and the layered structure of $\mathrm{MA}_{1+x} \mathrm{PbI}_{3+y}$ was proposed as the intermediate phase, similar to our observation of $\mathrm{MA}_{y} \mathrm{PbI}_{2.5-z}$.

To better understand the decomposition process from $\mathrm{MAPbI}_{3}$ to $\mathrm{PbI}_{2}$, we also investigate the intensity changes of diffraction spots during the decomposition as shown in Fig. $5 \mathrm{~m}$. Under the electron beam illumination, the intensities of the $\mathrm{MAPbI}_{3}(220)$, (110), and (200) spots decrease, indicating that the perovskite structure becomes defective. At $73 \mathrm{~s}$, the (100) superstructure reflection starts to appear likely due to the formation of $\mathrm{MAPbI}_{2.5}$. With the growing of $\mathrm{MAPbI}_{2.5}$, its (100) reflection intensity gradually increases. After a peak intensity of $\mathrm{MAPbI}_{2.5}$ (100) at $328 \mathrm{~s}, \mathrm{MAPbI}_{2.5}$ further loses I and MA to form $\mathrm{MA}_{y} \mathrm{PbI}_{2.5-z}(0 \leq y \leq 1$ and $0 \leq z \leq 0.5)$, leading to the reduction of superstructure reflection intensity. Meanwhile, the perovskite structure collapses and decomposes into a layered $\mathrm{PbI}_{2}$ structure. After $933 \mathrm{~s}$, the superstructure $\mathrm{MAPbI}_{2.5}$ has completely disappeared. And after $1524 \mathrm{~s}$, the reflections of $\mathrm{MA}_{y} \mathrm{PbI}_{2.5-z}(0 \leq y \leq$ 1 and $0 \leq z \leq 0.5)$ phase disappear and the intensity of the $(0 \overline{1} 4)$ spot of $\mathrm{PbI}_{2}$ is stabilized, indicating that the structure has completely decomposed into $\mathrm{PbI}_{2}$.

Finally, we investigate the effect of the dose rate on the degradation, and we determine the critical electron dose limit to obtain the intrinsic perovskite structure. Note that the perovskite framework remains until the $\mathrm{MA}_{y} \mathrm{PbI}_{2.5-z}(0 \leq y \leq 1$ and $0 \leq z \leq$ $0.5)$ collapses. Therefore, it is reasonable to expect that before the formation of $\mathrm{MAPbI}_{2.5}$ superstructure, the perovskite structure with a low density of iodine vacancies is robust and close to the ideal pristine structure. We find that typically the formation of

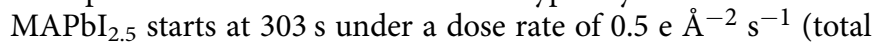



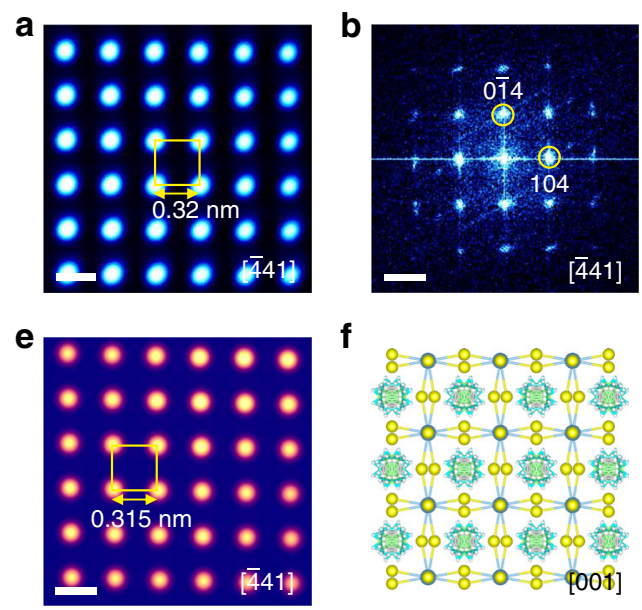
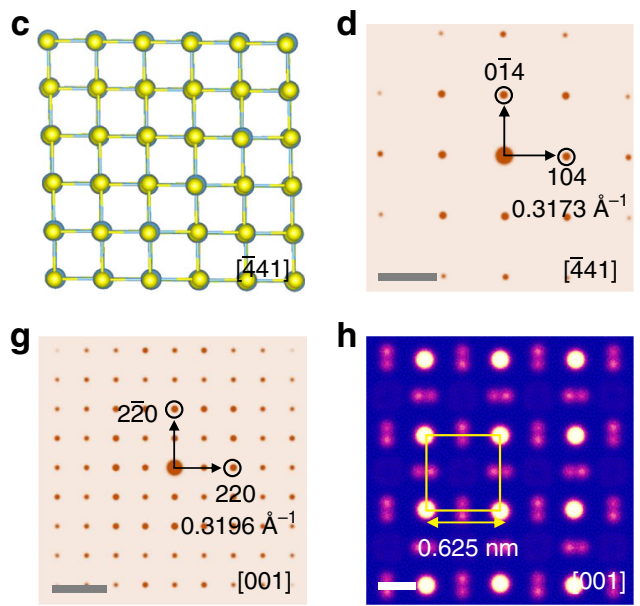

Fig. 3 Structure and ED difference between $\mathrm{Pbl}_{2}$ and tetragonal $\mathrm{MAPbl}_{3}$. a A STEM image of $\mathrm{Pbl}_{2}$ viewing along the [ $\left.\overline{4} 41\right]$ direction, judged from $\mathbf{b}$ the corresponding FFT pattern. $\mathbf{c}$ The atomic ball-and-stick model, $\mathbf{d}$ the simulated ED pattern, and e simulated STEM image of $\mathrm{Pbl}_{2}$ along the [4 441$]$ zone axis. f The atomic ball-and-stick model, $\mathbf{g}$ the simulated ED pattern, and $\mathbf{h}$ simulated STEM image of $\mathrm{MAPb}_{3}$ along the [001] zone axis. The ED patterns of Pbl 2 and $\mathrm{MAPb}_{3}$ are similar with a slight difference that the $\{2 \mathrm{~h}+1,2 \mathrm{~h}+1,0\}$ reflections [e.g., (110)] of $\mathrm{MAPbl}_{3}$ are not observed in that of $\mathrm{Pbl}{ }_{2}$. Besides, the distance between two brighter atoms is $0.625 \mathrm{~nm}$ for $\mathrm{MAPbl}_{3}$, which is about twice the distance between two brighter atoms $(0.315 \mathrm{~nm})$ of $\mathrm{Pbl}_{2} . \mathrm{Scale}$ bar, $0.3 \mathrm{~nm}(\mathbf{a}, \mathbf{e}, \mathbf{h}) ; 3 \mathrm{~nm}^{-1}(\mathbf{b}, \mathbf{d}, \mathbf{g})$

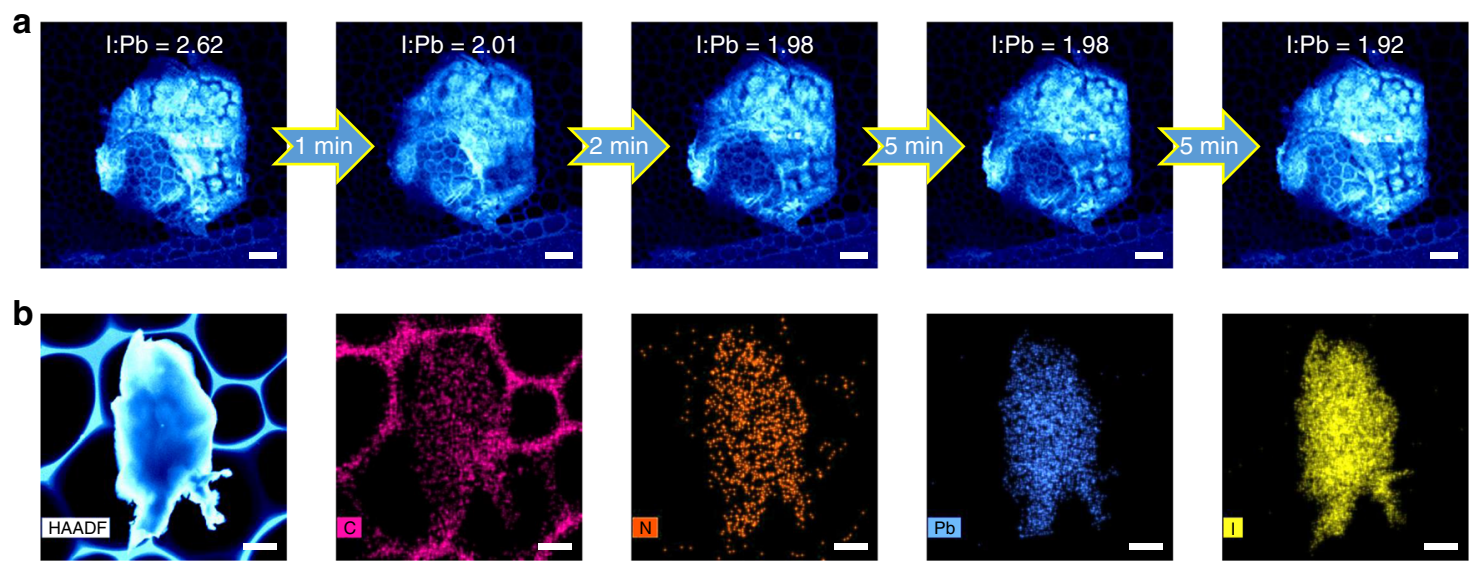

Fig. 4 The quantitative elemental analysis during the degradation of $\mathrm{MAPbl}_{3}$. a Scanning electron microscopy (SEM) images with the atomic ratio for I/Pb acquired from the corresponding energy-dispersive X-ray spectroscopy (EDX) mappings in Supplementary Fig. 10, showing that the atomic ratio of I/Pb gradually decreases to 2 . The beam was blank for a certain time indicated by the arrows after every spectrum was acquired. b A STEM image and the corresponding EDX mappings for the quantitative elemental analysis (Supplementary Table 2). Scale bar, $5 \mu \mathrm{m}(\mathbf{a}) ; 500 \mathrm{~nm}$ (b)

dose: 151 e $\AA^{-2}$ ) and $73 \mathrm{~s}$ under a dose rate of 1 e $\AA^{-2} \mathrm{~s}^{-1}$ (total dose: 73 e $\AA^{-2}$ ), while at 2 and 4 e $\AA^{-2} s^{-1}$, the decomposition has already begun at the first SAED images ( $20 \mathrm{~s}$ of exposure) (see details in Supplementary Fig. 13). Note that the decomposition not only depends on the total dose, but is also sensitive to dose rate, with higher rates resulting in accelerated decomposition under a similar total dose.

\section{Discussion}

The $\mathrm{MAPbI}_{3}$ is extremely sensitive to electron irradiation and thus it is easy to decompose into $\mathrm{PbI}_{2}$ accompanied by subtle changes in $\mathrm{ED}$, i.e., some of the diffraction spots are missing or weaken, or additional spots appear. Particularly, if we can only observe $\{2 \mathrm{~h}, 2 \mathrm{k}, 0\}$ diffraction spots along the [001] direction while the $\{2 \mathrm{~h}+1,2 \mathrm{k}+1,0\}$ reflections [e.g., (110)] are absent, it is highly likely that the perovskite structure has already decomposed into $\mathrm{PbI}_{2}$. However, many previous studies ignored such subtle changes and incorrectly identified the decomposed $\mathrm{PbI}_{2}$ to be
$\mathrm{MAPbI}_{3}$ based on the ED or FFT analysis. Consequently, extra attention must be paid during analyzing the electron microscopy data for these materials.

More importantly, we find that the decomposition of $\mathrm{MAPbI}_{3}$ is likely initiated with the loss of I ions to subsequently form superstructure $\mathrm{MAPbI}_{2.5}$, followed by the loss of MA and additional I ions to form $\mathrm{MA}_{y} \mathrm{PbI}_{2.5-z}(0 \leq y \leq 1$ and $0 \leq z \leq 0.5)$ accompanied by the disappearance of a superstructure, and finally decomposes into $\mathrm{PbI}_{2}$. Typically, under electron beam

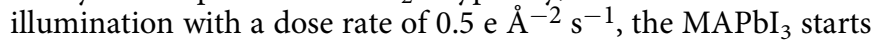
to change into $\mathrm{MAPbI}_{2.5}$ with ordered I vacancies after $303 \mathrm{~s}$, leaving sufficient time to acquire the ED for an intrinsic perovskite structure, while a higher dose rate results in faster decomposition under a similar total dose. With a dose rate higher than 2 e $\AA^{-2} s^{-1}$, decomposition has already begun at the first SAED images. These identified TEM conditions can be used to guide future electron microscopy characterization of these materials. 

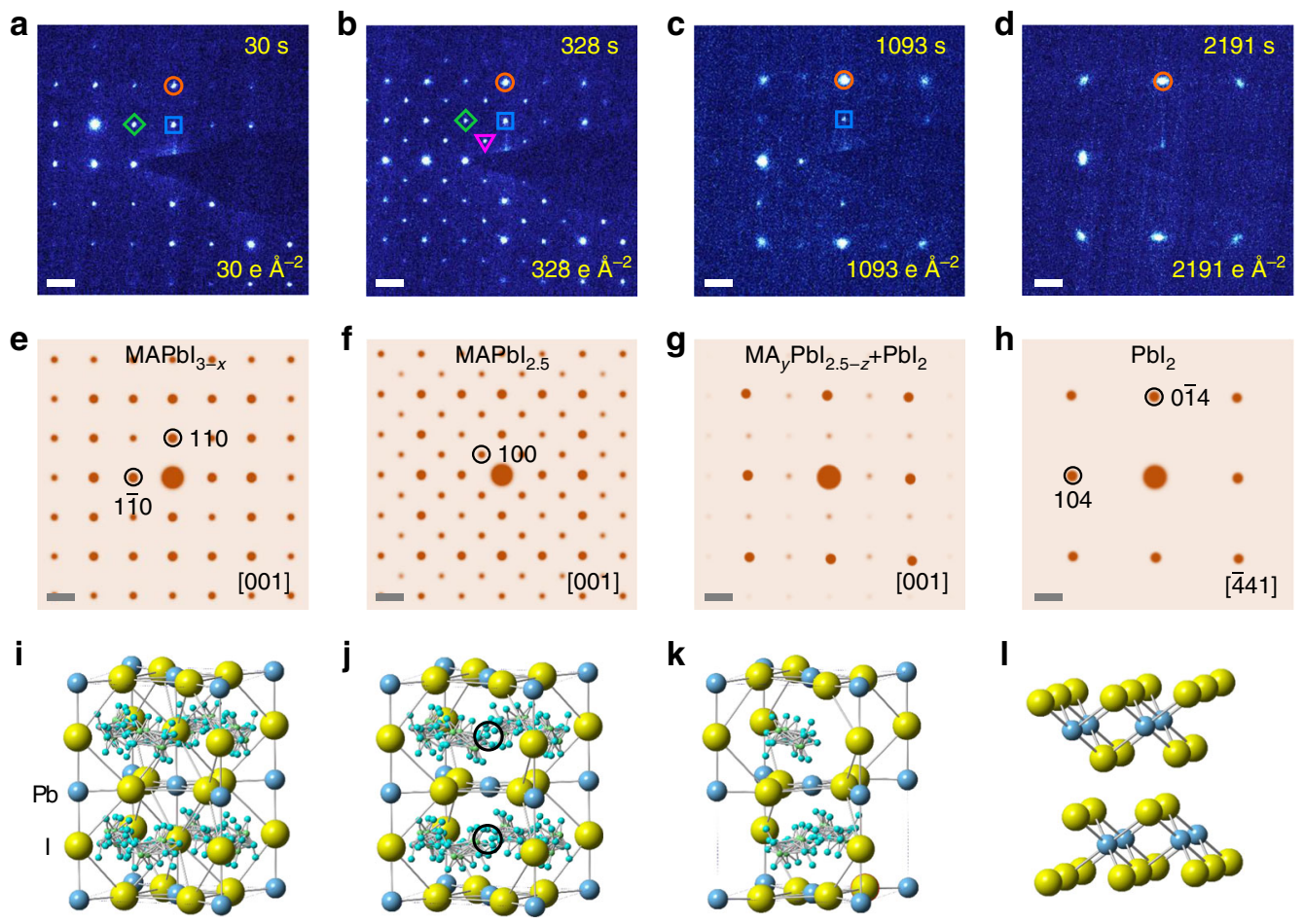

$\mathbf{k}$

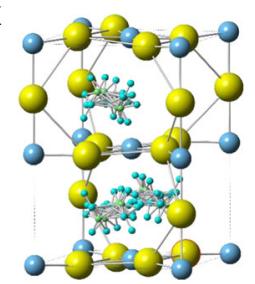

I

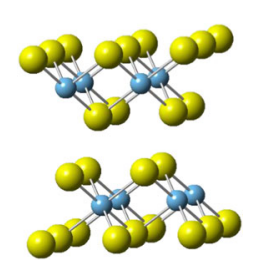

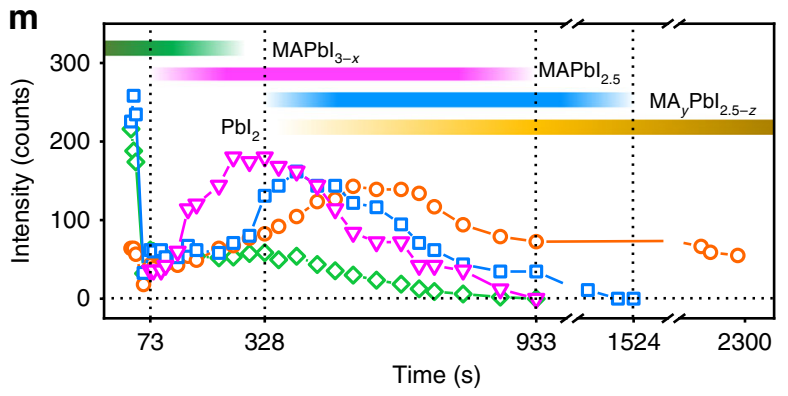

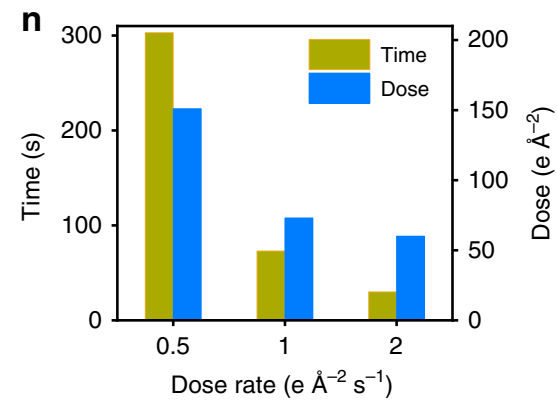

Fig. 5 The structural evolution during the decomposition from $\mathrm{MAPbl}_{3}$ to $\mathrm{Pbl}_{2}$. a-d Time-series SAED patterns showing that the structure decomposes from a $\mathrm{MAPb}_{3-x}[001]$ zone axis $(0<x<0.5)$, to $\mathbf{b}$ intermediate phase $\mathrm{MAPbl}_{2.5}, \mathbf{c} \mathrm{MA}_{y} \mathrm{Pbl}_{2.5-z}(0 \leq y \leq 1$ and $0 \leq z \leq 0.5)$, and $\mathbf{d} \mathrm{Pbl}_{2}[\overline{4} 41]$ zone axis sequentially under a dose rate of $1 \mathrm{e} \AA^{-2} \mathrm{~s}^{-1}$. The cumulative electron dose and time are labeled in each pattern. The blue squares mark the (110) reflection of $\mathrm{MAPbl}_{3}$ in $\mathbf{a}, \mathrm{MAPbl}_{2.5}$ in $\mathbf{b}$, and $\mathrm{MAPbl}_{2.5}$ and $\mathrm{MA}_{y} \mathrm{Pbl}_{2.5-z}$ in $\mathbf{c}$. The green diamonds mark the (200) reflection of $\mathrm{MAPbl}_{3}$ in $\mathbf{a}$, and $\mathrm{MAPbl}_{2.5}$ in $\mathbf{b}$. The orange circles mark the (220) reflection of $\mathrm{MAPbl}_{3}$ in $\mathbf{a}, \mathrm{MAPbl}_{2.5}$ in $\mathbf{b}, \mathrm{MAPbl}_{2.5}$ and $\mathrm{MA}_{y} \mathrm{Pbl}_{2.5-z}$ in $\mathbf{c}$, and $(0 \overline{1} 4)$ of $\mathrm{Pbl}_{2}$ in $\mathbf{d}$. The pink triangles mark the (100) reflection of $\mathrm{MAPbl}_{2.5}$ in $\mathbf{b}$. e-h The corresponding simulated ED patterns of e $\mathrm{MAPbl}_{3}, \mathbf{f} \mathrm{MAPbl}_{2.5}, \mathbf{g} 35 \% \mathrm{MA}_{y} \mathrm{Pbl}_{2.5-z}+65 \% \mathrm{Pbl}_{2}$ (see Supplementary Fig. 12), and $\mathbf{h} \mathrm{Pbl}_{2}$. Note that $\mathrm{MA}_{y} \mathrm{Pbl}_{2.5-z}(0 \leq y \leq 1$ and $0 \leq z \leq 0.5)$ with random vacancies should have the same ED pattern with $\mathrm{MA}_{y} \mathrm{Pbl}_{2.5-z}(y=0$ and $z=0.5)$ without considering the very diffused reflections from random vacancies. $\mathbf{i}-\mathbf{I}$ The atomistic structures of $\mathbf{i}$ MAPbl 3 , $\mathbf{j} \mathrm{MAPbl}_{2.5}, \mathbf{k} \mathrm{MA}_{y} \mathrm{Pbl}_{2.5-z}(0 \leq y \leq 1$ and $0 \leq z \leq 0.5)$, and $\mathbf{I} \mathrm{Pbl}_{2}$. The black circles in $\mathbf{j}$ represent the ordered iodine vacancies. $\mathbf{m}$ The diffraction intensity is plotted as a function of time (total dose). The pink triangles, orange circles, blue squares, and green diamonds correspond to those label shapes in a-d. The green, pink, blue, and orange color bars roughly estimate the existence of $\mathrm{MAPb}_{3-x}, \mathrm{MAPbl}_{2.5}, \mathrm{MA}_{y} \mathrm{Pbl}_{2.5-z}$, and $\mathrm{Pbl}_{2}$ at different stages. Note that the values of the intensities for (110) (blue squares) and (100) (pink triangles) spots are set to be 1.8 and 6 times of the original values for comparison. $\mathbf{n}$ During the decomposition of the perovskite at different dose rates, the measured critical electron dose and time for the appearance of a superstructure. Scale bar, $1 \mathrm{~nm}^{-1}(\mathbf{a}-\mathbf{h})$

Moreover, considering the similarities between electron beam illumination and optical illumination, as well as the heating and electric field, during which the ions gain energy to overcome the migration barriers to trigger the phase transitions, we believe that the electro-driven ion migration and heating will likely lead to a similar structural degradation process, which may be responsible for the poor long-term device stability. In this sense, the observed decomposition of perovskite under the electron beam illumination can help us to understand the structure instability issue for these materials under device operation, which is currently under further investigation.

\section{Methods}

MAPbl $_{3}$ synthesis. The single-crystal perovskite $\mathrm{MAPbI}_{3}$ was synthesized as follows. Two $\mathrm{FTO} / \mathrm{TiO}_{2}$ substrates were clamped together using the fixed-size card slots. In total, $2 \mathrm{~mL}$ of precursor solution of $\mathrm{PbI}_{2} / \mathrm{MAI}$ (molar ratio 1:1) was prepared in $\gamma$-butyrolactone. After $12 \mathrm{~h}$ of continuous stirring, a homogeneous $\mathrm{MAPbI}_{3}$ solution at a concentration of $1.3 \mathrm{~mol} / \mathrm{L}$ was obtained, and then filtered using a polytetrafluoroethylene (PTFE) filter with $0.22-\mu \mathrm{m}$ pore size. The clamped $\mathrm{FTO} / \mathrm{TiO}_{2}$ substrates were then vertically dipped into a $10-\mathrm{mL}$ beaker containing $\mathrm{MAPbI}_{3}$ precursor solution kept on a hot plate at $120^{\circ} \mathrm{C}$ in nitrogen atmosphere, and the feeding precursor solution was added every $12 \mathrm{~h}$. After a certain period of 27 days, the substrates with a self-grown film were taken out, and then dried at $120^{\circ} \mathrm{C}$ for $10 \mathrm{~min}$ in nitrogen glovebox. 
Data acquisition and analysis. Powder XRD patterns were obtained on D8 Advance diffractometer using $\mathrm{Cu}$ Ka radiation $(40 \mathrm{kV}$ and $40 \mathrm{~mA})$ with a scanning rate of $4^{\circ} \mathrm{min}^{-1}$ for wide-angle test increment. The morphologies of the sample were examined by SEM (ZEISS Gemini SEM 300), and the SEM-EDX was carried out at $10 \mathrm{kV}$ with $1 \mathrm{~min}$ for acquiring the spectra, using a current of $\sim 1 \mathrm{nA}$. The beam was kept blank for several minutes after each spectrum was acquired because the sample is sensitive to the electron beam.

Single-crystal X-ray diffraction was carried out at Sector 7-ID-C, Advanced Photon Source, Argonne National Laboratory, using $10-\mathrm{keV}$ X-ray with a $300 \times$ $300 \mu^{2}$ beam size as defined by a slit. A Huber six-circle diffractometer coupled with a PILATUS $100-\mathrm{K}$ area detector was employed for measurements of the single-crystalline samples. The detector was placed downstream from the samples such that $\sim 8^{\circ}$ coverage in the $2 \theta$-angle and $\sim 3^{\circ}$ in the $\chi$-angle was obtained. Rocking ( $\omega$-angle) scans around each reflection were recorded and for the data presented, the resultant $3 \mathrm{D}$ data volume was reduced by integrating the rocking angles.

High-resolution TEM, HAADF STEM images were acquired at an aberrationcorrected FEI (Titan Cubed Themis G2) operated at $80 \mathrm{kV}$ equipped with an XFEG gun and Bruker Super-X EDX detectors. STEM images were acquired with a beam current of 6-10 pA, a convergence semi-angle of $25 \mathrm{mrad}$, and a collection semi-angle snap in the range of 53-260 mrad. The STEM-EDX mapping was obtained with a beam current of $1 \mathrm{nA}$ and counts ranging from $2 \mathrm{k}$ cps to $8 \mathrm{k}$ cps for $\sim 5$ min. The SAED patterns for Fig. 5, Supplementary Fig. 11, and Fig. 13 were acquired at $300 \mathrm{kV}$

Under the STEM mode for Figs. 2 and 3, the dose rate is estimated to be 220-360 e $\AA^{-2} \mathrm{~s}^{-1}$ which is calculated by dividing the screen current $(6 \sim 10 \mathrm{pA})$ by the area of the raster ${ }^{49}$. To get a satisfactory STEM image, we need to carefully align the zone axis and adjust the astigmatism and the focus before image acquisition, which is time-consuming and costs about 5-10 min. To record the SAED patterns of Fig. 1d, the sample is always observed at low magnifications (10000-30000) and the dose rate is estimated to be $100-300$ e $\AA^{-2} \mathrm{~s}^{-1}$. It usually takes $30-100 \mathrm{~s}$ to record a SAED image after the sample comes into sight. Under these conditions, judging from the data we acquired, the samples were decomposed due to large electron doses. To obtain the ideal pristine structure, we make the dose rate as low as possible. For Fig. 5, Supplementary Figs. 11 and 13, the dose rate ranges from 0.5 to 4 e $\AA^{-2} s^{-1}$.

The simulations of the ED pattern were performed by using Crystalmaker software, and the ball-and-stick models were formed using Vesta software. The FFT and inverse FFT patterns were obtained using DigitalMicrograph (Gatan) software. HAADF images in Fig. 2, Fig. 3, and Supplementary Fig. 5 were averaged from multiple regions with a homemade MATLAB code to reduce noise. The plots were drawn using Origin 2018.

STEM simulation. STEM simulation was carried out by Kirkland with COMPUTEM software ${ }^{50}$. Specifically, the technique involves dividing the sample into a number of thin slices normal to the incident electron beam and calculating the contribution to the cross-section at each slice. First, we used Vesta software to construct a $15 \times 15 \times 5$ supercell containing 12,352 atoms based on the $\mathrm{PbI}_{2} \mathrm{CIF}$ file $\mathrm{e}^{39}$ and a $5 \times 5 \times 5$ supercell containing 41,387 atoms from the $\mathrm{MAPbI}_{3} \mathrm{CIF}$ file ${ }^{51}$. Then a homemade MATLAB code was used to rotate the structure to the desired zone axes. The STEM simulated parameters were set with the beam energy of $80 \mathrm{keV}$ (accelerating voltage), object aperture of $25.1 \mathrm{mrad}$ (convergence semiangle), and a STEM ADF detector of 53-260 mrad (collection semi-angle). Besides, the transmission function size was $2048 \times 2048$ pixels and STEM probe size was $512 \times 512$ pixels. The other parameters were set as default. To achieve realistic computing time for these configurations, a limited area was chosen to run the STEM simulations. The thickness of the sample along different zone axes was estimated to range from 4.5 to $6.5 \mathrm{~nm}$.

Ab initio simulation. All ab initio simulations were performed using Vienna $\mathrm{Ab}$ Initio Simulation Package ${ }^{52-54}$ projected augmented wave $(\mathrm{PAW})^{55,56}$ potential. For calculations of $\mathrm{PbI}_{2}$, strongly constrained and appropriately normed (SCAN) ${ }^{57}$ exchange-correlation functional was adopted. Plane-wave cutoff of $650 \mathrm{eV}$ was used. For structural optimization, $6 \times 6 \times 6 \mathrm{k}$-mesh was used. For molecular dynamics simulations, $1 \times 1 \times 1 \mathrm{k}$-mesh and 81 -atom supercell were used. Isothermal-isobaric ensemble (NPT) $)^{58}$ simulations at $300 \mathrm{~K}$ were run 5000 steps to obtain averaged lattice constants and subsequently, canonical ensemble (NVT) simulations at the same temperature were run 10,000 steps using Nosé-Hoover thermostat ${ }^{59,60}$, of which the final 8000 steps were used to average the atomic positions. The time step was chosen to be 2 fs for both simulations and the energy drift was less than $1 \mathrm{meV}(\mathrm{ps})^{-1}$ per atom for NVT simulations.

\section{Data availability}

The authors declare that all relevant data are included in the paper and its Supplementary Information files. Additional data including the codes are available from the corresponding author upon reasonable request.
Received: 26 April 2018 Accepted: 19 October 2018

Published online: 15 November 2018

\section{References}

1. Li, X. et al. A vacuum flash-assisted solution process for high-efficiency largearea perovskite solar cells. Science 353, 58-62 (2016).

2. Chen, W. et al. Efficient and stable large-area perovskite solar cells with inorganic charge extraction layers. Science 350, 944 (2015).

3. Burschka, J. et al. Sequential deposition as a route to high-performance perovskite-sensitized solar cells. Nature 499, 316-319 (2013).

4. Miyasaka, T., Teshima, A., Shirai, K., Y. \& Miyasaka, T. Organometal halide perovskites as visible-light sensitizers for photovoltaic cells. J. Am. Chem. Soc. 131, 6050-6051 (2009).

5. National Renewable Energy Laboratory (NREL). Efficiency Records Chart. https://www.nrel.gov/pv/assets/pdfs/pv-efficiencies-07-17-2018.pdf (Accessed on 15 October 2018).

6. Divitini, G. et al. In situ observation of heat-induced degradation of perovskite solar cells. Nat. Energy 1, 15012 (2016).

7. Aristidou, N. et al. Fast oxygen diffusion and iodide defects mediate oxygeninduced degradation of perovskite solar cells. Nat. Commun. 8, 15218 (2017).

8. Yang, J., Siempelkamp, B. D., Liu, D. \& Kelly, T. L. Investigation of $\mathrm{CH}_{3} \mathrm{NH}_{3} \mathrm{PbI}_{3}$ degradation rates and mechanisms in controlled humidity environments using in situ techniques. ACS Nano 9, 1955-1963 (2015).

9. Bryant, D. et al. Light and oxygen induced degradation limits the operational stability of methylammonium lead triiodide perovskite solar cells. Energy Environ. Sci. 9, 1655-1660 (2016).

10. Li, C. et al. Iodine migration and its effect on hysteresis in perovskite solar cells. Adv. Mater. 28, 2446-2454 (2016).

11. Yang, D., Ming, W., Shi, H., Zhang, L. \& Du, M. Fast diffusion of native defects and impurities in perovskite solar cell material $\mathrm{CH}_{3} \mathrm{NH}_{3} \mathrm{PbI}_{3}$. Chem. Mater. 28, 4349-4357 (2016)

12. Ito, S., Tanaka, S., Manabe, K. \& Nishino, H. Effects of surface blocking laye of $\mathrm{Sb}_{2} \mathrm{~S}_{3}$ on nanocrystalline $\mathrm{TiO}_{2}$ for $\mathrm{CH}_{3} \mathrm{NH}_{3} \mathrm{PbI}_{3}$ perovskite solar cells. J. Phys. Chem. C. 118, 16995-17000 (2014).

13. Yang, B. et al. Enhancing ion migration in grain boundaries of hybrid organicinorganic perovskites by chlorine. Adv. Funct. Mater. 27, 1700749 (2017).

14. Eames, C. et al. Ionic transport in hybrid lead iodide perovskite solar cells Nat. Commun. 6, 7497 (2015)

15. Anaya, M. et al. Strong quantum confinement and fast photoemission activation in $\mathrm{CH}_{3} \mathrm{NH}_{3} \mathrm{PbI}_{3}$ perovskite nanocrystals grown within periodically mesostructured films. Adv. Opt. Mater. 5, 1601087 (2017).

16. Yang, $M$. et al. Square-centimeter solution-processed planar $\mathrm{CH}_{3} \mathrm{NH}_{3} \mathrm{PbI}_{3}$ perovskite solar cells with efficiency exceeding 15\%. Adv. Mater. 27, 6363-6370 (2015)

17. Xiao, M. et al. A fast deposition-crystallization procedure for highly efficient lead iodide perovskite thin-film solar cells. Angew. Chem. Int. Ed. 53, 9898-9903 (2014).

18. Zhang, D. et al. Atomic-resolution transmission electron microscopy of electron beam-sensitive crystalline materials. Science 359, 675-679 (2018).

19. Ji, F. et al. Simultaneous evolution of uniaxially oriented grains and ultralowdensity grain-boundary network in $\mathrm{CH}_{3} \mathrm{NH}_{3} \mathrm{PbI}_{3}$ perovskite thin films mediated by precursor phase metastability. ACS Energy Lett. 2, 2727-2733 (2017).

20. Zhao, C. et al. Diffusion-correlated local photoluminescence kinetics in $\mathrm{CH}_{3} \mathrm{NH}_{3} \mathrm{PbI}_{3}$ perovskite single-crystalline particles. Sci. Bull. 61, 665-669 (2016).

21. Li, D. et al. Size-dependent phase transition in methylammonium lead iodide perovskite microplate crystals. Nat. Commun. 7, 11330 (2016)

22. Zhou, Y. et al. Crystal morphologies of organolead trihalide in mesoscopic/ planar perovskite solar cells. J. Phys. Chem. Lett. 6, 2292-2297 (2015).

23. Yang, B. et al. Controllable growth of perovskite films by room-temperature air exposure for efficient planar heterojunction photovoltaic cells. Angew. Chem. Int. Ed. 54, 14862-14865 (2015)

24. Long, $\mathrm{M}$. et al. Textured $\mathrm{CH}_{3} \mathrm{NH}_{3} \mathrm{PbI}_{3}$ thin film with enhanced stability for high performance perovskite solar cells. Nano Energy 33, 485-496 (2017).

25. Son, D. et al. Self-formed grain boundary healing layer for highly efficient $\mathrm{CH}_{3} \mathrm{NH}_{3} \mathrm{PbI}_{3}$ perovskite solar cells. Nat. Energy 1, 16081 (2016).

26. Kollek, T. et al. Porous and shape-anisotropic single crystals of the semiconductor perovskite $\mathrm{CH}_{3} \mathrm{NH}_{3} \mathrm{PbI}_{3}$ from a single-source precursor. Angew. Chem. Int. Ed. 54, 1341-1346 (2015).

27. Gao, L. et al. Passivated single-crystalline $\mathrm{CH}_{3} \mathrm{NH}_{3} \mathrm{PbI}_{3}$ nanowire photodetector with high detectivity and polarization sensitivity. Nano. Lett. 16, 7446-7454 (2016).

28. Zhu, F. et al. Shape evolution and single particle luminescence of organometal halide perovskite nanocrystals. ACS Nano 9, 2948-2959 (2015).

29. Zhu, H. et al. Lead halide perovskite nanowire lasers with low lasing thresholds and high quality factors. Nat. Mater. 14, 636-642 (2015). 
30. Kim, T. W. et al. Self-organized superlattice and phase coexistence inside thin film organometal halide perovskite. Adv. Mater. 30, 1705230 (2018).

31. Rothmann, M. U. et al. Direct observation of intrinsic twin domains in tetragonal $\mathrm{CH}_{3} \mathrm{NH}_{3} \mathrm{PbI}_{3}$. Nat. Commun. 8, 14547 (2017).

32. Gao, P. et al. Electrically driven redox process in cerium oxides. J. Am. Chem. Soc. 132, 4197-4201 (2010).

33. Ishikawa, R. et al. Direct observation of dopant atom diffusion in a bulk semiconductor crystal enhanced by a large size mismatch. Phys. Rev. Lett. 113, 155501 (2014).

34. Egerton, R. F., Li, P. \& Malac, M. Radiation damage in the TEM and SEM. Micron 35, 399-409 (2004).

35. Zhao, J. et al. Single crystalline $\mathrm{CH}_{3} \mathrm{NH}_{3} \mathrm{PbI}_{3}$ self-grown on $\mathrm{FTO} / \mathrm{TiO}_{2}$ substrate for high efficiency perovskite solar cells. Sci. Bull. 62, 1173-1176 (2017).

36. Huang, B. et al. Ferroic domains regulate photocurrent in single-crystalline $\mathrm{CH}_{3} \mathrm{NH}_{3} \mathrm{PbI}_{3}$ films self-grown on $\mathrm{FTO} / \mathrm{TiO}_{2}$ substrate. npj Quantum Mater. 3, 30 (2018).

37. Ye, T. et al. Single-crystalline lead halide perovskite arrays for solar cells. J. Mater. Chem. A 4, 1214-1217 (2016).

38. Baikie, T. et al. Synthesis and crystal chemistry of the hybrid perovskite $\mathrm{CH}_{3} \mathrm{NH}_{3} \mathrm{PbI}_{3}$ for solid-state sensitised solar cell applications. J. Mater. Chem. A 1, 5628 (2013).

39. Wyckoff, R. W. Crystal Structure 2nd edn, Vol. 1, 239-444 (Interscience Publishers Press, New York, 1993).

40. Jeangros, Q. et al. In situ TEM analysis of organic-inorganic metal-halide perovskite solar cells under electrical bias. Nano. Lett. 16, 7013-7018 (2016).

41. Yang, B. et al. Observation of nanoscale morphological and structural degradation in perovskite solar cells by in situ TEM. ACS Appl. Mater. Inter. 8, 32333-32340 (2016).

42. Haruyama, J., Sodeyama, K., Han, L. \& Tateyama, Y. First-principles study of ion diffusion in perovskite solar cell sensitizers. J. Am. Chem. Soc. 137, 10048-10051 (2015)

43. Azpiroz, J. M., Mosconi, E., Bisquert, J. \& De Angelis, F. Defect migration in methylammonium lead iodide and its role in perovskite solar cell operation. Energy Environ. Sci. 8, 2118-2127 (2015).

44. Cherry, M., Islam, M. S. \& Catlow, C. R. A. Oxygen ion migration in perovskite-type oxides. J. Solid State Chem. 118, 125-132 (1995).

45. Minns, J. L., Zajdel, P., Chernyshov, D., van Beek, W. \& Green, M. A. Structure and interstitial iodide migration in hybrid perovskite methylammonium lead iodide. Nat. Commun. 8, 15152 (2017).

46. Fernandez-Moran, H., Ohstuki, M., Hibino, A. \& Hough, C. Electron microscopy and diffraction of layered, superconducting intercalation complexes. Science 174, 498-500 (1971).

47. Safran, S. A. \& Hamann, D. R. Long-range elastic interactions and staging in graphite intercalation compounds. Phys. Rev. Lett. 42, 1410-1413 (1979).

48. Pellegrino, G. et al. From $\mathrm{PbI}_{2}$ to $\mathrm{CH}_{3} \mathrm{NH}_{3} \mathrm{PbI}_{3}$ through layered intermediates. J. Phys. Chem. C. 120, 19768-19777 (2016).

49. Johnston-Peck, A. C., DuChene, J. S., Roberts, A. D., Wei, W. D. \& Herzing, A. A. Dose-rate-dependent damage of cerium dioxide in the scanning transmission electron microscope. Ultramicroscopy 170, 1-9 (2016).

50. Kirkland, E. J. Advanced Computing in Electron Microscopy. (Plenum Press, New York, 1998).

51. Yamada, Y. et al. Dynamic optical properties of $\mathrm{CH}_{3} \mathrm{NH}_{3} \mathrm{PbI}_{3}$ single crystals as revealed by one- and two-photon excited photoluminescence measurements. J. Am. Chem. Soc. 137, 10456-10459 (2015).

52. Kresse, G. \& Furthmüller, J. Efficiency of ab-initio total energy calculations for metals and semiconductors using a plane-wave basis set. Comp. Mater. Sci. 6, 15-50 (1996).

53. Furthmüller, J. \& Kresse, G. Efficient iterative schemes for ab initio totalenergy calculations using a plane-wave basis set. Phys. Rev. B 54, 11169-11186 (1996).

54. Hafner, J. \& Kresse, G. Ab initio molecular dynamics for liquid metals. Phys. Rev. B 47, 558-561 (1993).

55. Joubert, D. \& Kresse, G. From ultrasoft pseudopotentials to the projector augmented-wave method. Phys. Rev. B 59, 1758-1775 (1999).
56. Jepsen, O., Andersen, O. K. \& Blöchl, P. E. Improved tetrahedron method for Brillouin-zone integrations. Phys. Rev. B 49, 16223-16233 (1994).

57. Marsman, M. et al. Self-consistent meta-generalized gradient approximation within the projector-augmented-wave method. Phys. Rev. B 84, 035117 (2011).

58. Hernández, E. Metric-tensor flexible-cell algorithm for isothermal-isobaric molecular dynamics simulations. J. Chem. Phys. 115, 10282-10290 (2001).

59. Nosé, S. A unified formulation of the constant temperature molecular dynamics methods. J. Chem. Phys. 81, 511-519 (1984).

60. Hoover, W. G. Canonical dynamics: equilibrium phase-space distributions. Phys. Rev. A. 31, 1695-1697 (1985).

\section{Acknowledgements}

The work was supported by the National Key R\&D Program of China [grant numbers 2016YFA0300804, 2016YFA0300903, and 2016YFA0201001]; National Natural Science Foundation of China [grant numbers 51502007, 51672007, 51575135, U1537206, 11772207, and 11327902]; National Equipment Program of China [grant number ZDYZ2015-1], and "2011 Program" Peking-Tsinghua-IOP Collaborative Innovation Center of Quantum Matter. The authors acknowledge Electron Microscopy Laboratory in Peking University for the use of Cs-corrected electron microscope. We are grateful for computational resources provided by TianHe-1A in Tianjin and the High-performance Computing Platform of Peking University.

\section{Author contributions}

P.G., J.L., J.Q., J.Z., and X.L. conceived and supervised the project. S.C. performed the experiment and analyzed experimental data with the direction of P.G. and help from J.Z., K.L., and D.Y.; J.Z., G.K., and Y.Z. grew the crystals and carried out the XRD measurement; S.C., N.X., J.Z., and G.K. did the SEM measurement; X.Z. conducted the ab initio simulation with the guidance of X.L.; Q.L. did and analyzed the synchrotron XRD; N.L., S.C., and Y.Y. carried out the STEM simulations. Q.Z. J.C., J.F., and J.Q. provided crystals. S.C., J.L., and P.G. wrote the manuscript and all authors participated in the revision.

\section{Additional information}

Supplementary Information accompanies this paper at https://doi.org/10.1038/s41467018-07177-y.

Competing interests: The authors declare no competing interests.

Reprints and permission information is available online at http://npg.nature.com/ reprintsandpermissions/

Publisher's note: Springer Nature remains neutral with regard to jurisdictional claims in published maps and institutional affiliations.

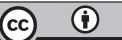

Open Access This article is licensed under a Creative Commons Attribution 4.0 International License, which permits use, sharing, adaptation, distribution and reproduction in any medium or format, as long as you give appropriate credit to the original author(s) and the source, provide a link to the Creative Commons license, and indicate if changes were made. The images or other third party material in this article are included in the article's Creative Commons license, unless indicated otherwise in a credit line to the material. If material is not included in the article's Creative Commons license and your intended use is not permitted by statutory regulation or exceeds the permitted use, you will need to obtain permission directly from the copyright holder. To view a copy of this license, visit http://creativecommons.org/ licenses/by/4.0/.

(C) The Author(s) 2018 